\title{
Studies on Microbial Dynamics in Little Millet (Panicum sumatrense L.) based Intercropping System under Rainfed Condition
}

\author{
S. Keerthanapriya ${ }^{1}$, M. Hemalatha ${ }^{1}$, S.P. Ramanathan ${ }^{1}$ and B. Jeberlin Prabina ${ }^{2}$ \\ ${ }^{1}$ Department of Agronomy, ${ }^{2}$ Department of SS\&AC, Agricultural College\& Research Institute, \\ Killikulam, Thoothukudi-628252, Tamil Nadu, India \\ *Corresponding author
}

\section{A B S T R A C T}

Keywords

Little millet, intercropping, Bacteria, Fungi, Actinobacteria, Azospirillum, Dehydrogenase enzyme activity

Article Info

Accepted:

07 May 2019

Available Online:

10 June 2019
A field experiment was conducted at Agricultural College and Research Institute, Killikulam during rabi season (October 2018 - January 2019) to evolve suitable little millet (Panicum sumatrense L) based intercropping system under rainfed condition. The experiment was laid out in randomized block design with eight treatments and replicated thrice. The population of soil bacteria, fungi, actinobacteria, Azospirillum and dehydrogenase enzyme activities were recorded under different little millet based intercropping system as a measure of soil microbial dynamics. Among the treatments, soil microbial population was higher in little millet intercropped with both pulses and legumes. Also, the dehydrogenase enzyme activity was more in intercropping compared to mono-cropping and nonlegume intercropping system.

\section{Introduction}

India is the largest producer of variety of millets, which are often referred as coarse cereals. Realizing the nutrient richness of these grains they are now considered as "Nutri-cereals". In India, little millet holds an area of 2.25 lakh ha with a production of 1.59 lakh tonnes and productivity of $707 \mathrm{~kg} / \mathrm{ha}$ under small millet (Anonymous, 2016). Little millet is a good source of protein for vegetarians. Little millet is used to cure cancer, diabetes, asthma, high blood pressure and provides relief from constipation, bloating and cramps. Little millet is used mainly as rice or bread, which serve as the poor man's food. The area under millets are shrinking over years due to replacement of little millet crop with more remunerative crops. Intercropping system has been recognized as beneficial cropping system under rain-fed condition and intercropping with suitable crops provide a better option for farmers. Cultivation of small millets in 
general and more specifically little millet can be made more remunerative by addition of certain high value intercrops.

Intercropping increases soil microbial, soil nutrients, soil enzymes and biochemical properties. Ahmad et al., 2013 recorded enhanced soil microbial activity in pepper with green garlic intercropping system. Li et al., 2016 reported increased soil enzymatic activities in Maize + peanut intercropping system. Intercropping of Baby corn + okra system enhanced the actinomycetes population (Rekha et al., 2017). Enhanced soil microbial diversity was observed in Trifolium-cucumber and mustard-cucumber system (Li et al., 2018). Mulberry + alfalfa intercropping enhanced soil bacterial community, due to increasing soil total carbon, available phosphate and available potassium (Zhang et al., 2017). Bt cotton + fodder cowpea intercropping system increased bacterial population and $\mathrm{Bt}$ cotton + moth bean enhanced fungus and actinomycetes population (Daisy et al., 2018).

Intercropping system not only the influences soil characteristics but also have positive impact on economics. Dubey et al., 2001 reported little millet intercropped with pigeon pea gives higher benefit cost ratio of 1.83 .
Little millet + green gram resulted in a benefit cost ratio of 2.42 (Sharmili and Manoharan, 2018). Higher returns and benefit cost ratio of 3.73 were recorded in foxtail millet + pigeon pea intercropping system (Himasree et al., 2017).

In Tamirabarani command area, rice followed by pulses is the practice commonly followed and intercropping system is not prevalent. Also, suitable intercropping system is not evolved for Tamirabarani command area. Since, cultivation of legumes and pulses, improves soil physical properties, soil nitrogen content, microbial activity and restores organic matter content; vegetables form part of daily diet, intercropping of little millets with legumes, pulses and vegetables were chosen for the study to have nutritional security and to generate additional income to the farmers of Tamirabarani command area.

\section{Materials and Methods}

The experimental field is geographically located in the Southern part of Tamil Nadu at $8^{\circ} 46^{\prime} \mathrm{N}$ latitude and $77^{\circ} 42^{\prime} \mathrm{E}$ longitude with an altitude of $40 \mathrm{~m}$ above MSL. The experiment was laid out in randomized block design with three replications with the following treatments

$$
\begin{array}{ll}
\mathrm{T}_{1} & \text { Little millet sole crop } \\
\mathrm{T}_{2} & \text { Little millet + Blackgram }(4: 1) \\
\mathrm{T}_{3} & \text { Little millet + Greengram }(4: 1) \\
\mathrm{T}_{4} & \text { Little millet + Cowpea }(4: 1) \\
\mathrm{T}_{5} & \text { Little millet + Groundnut }(4: 1) \\
\mathrm{T}_{6} & \text { Little millet + Horsegram }(4: 1) \\
\mathrm{T}_{7} & \text { Little millet + Small onion }(4: 1) \\
\mathrm{T}_{8} & \text { Little millet + Coriander }(4: 1)
\end{array}
$$

\section{Soil sampling}

Soil samples of black gram, green gram, cowpea, groundnut, horse gram, small onion and coriander were collected during different plant growth period as a $20 \mathrm{~cm}^{2}$ by $30 \mathrm{~cm}$ depth of soil. Plants and soil samples were immediately transferred in polyethylene bags to avoid excessive desiccation during transport and were stored thereafter at $4^{\circ} \mathrm{C}$. 
Each sample was separated into two fractions: bulk soil (NRS) and rhizosphere soil (RS).

In order to collect bulk soil, plants were vigorously shaken by hand for ten minutes (Figure 1). Rhizosphere soil was afterwards collected by hand shaking roots for 10 minutes in one litre of sterile $0.9 \% \mathrm{NaCl}$ solution to remove the adhering soil (Figure 2).

\section{Enumeration of total bacteria, fungi and actinobacteria}

The soil samples were serially diluted to enumerate the total bacteria, fungi and actinobacteria of both rhizosphere and nonrhizosphere soil. The population of each group was calculated and expressed as cfu per $\mathrm{g}$ of dry weight soil.

\section{Serial dilution and plating technique}

One gram of soil sample was weighed and added to $100 \mathrm{ml}$ water blank. These samples were shaken for 15 minutes to get complete dispersion (This gives $10^{-2}$ dilution). One $\mathrm{ml}$ of the suspension were transferred to nineml water blank (This gives $10^{-3}$ dilution). Transferred serially upto $10^{-8}$ dilution or required dilution. One $\mathrm{ml}$ of appropriate dilutions was transferred to petri dishes. Three replications for each dilution were maintained. Media was melted and cooled of about $15-20 \mathrm{ml}$ was poured and mixed well by rotating clock wise and anti-clock wise for 3 or 4 times and allowed it for complete solidification. The plates were incubated in inverted position at room temperature or in an incubator for 2-7 days. The bacteria, fungi and actinobacteria colonies were observed after 2 days, 3-4 days and 7 days respectively. The colonies per plate were counted and the soil microbial population was calculated. Each colony is referred as colony forming units (cfu).
(Note: Normally $10^{-7}$ or $10^{-8}$ dilution can be used for bacteria; $10^{-3}$ or $10^{-4}$ dilution for actinobacteria and $10^{-4}$ or $10^{-5}$ dilution for fungi can be used if soil is used for enumeration).

\section{Enumeration of Azospirillum}

The population of Azospirillum was enumerated from soil using Most Probable Number (MPN) method. This method relies upon the pattern of positive and negative growth of Azospirillum in the tubes inoculated with a consecutive series of dilutions of the soil / root sample. Based on the number of positive tubes, the population estimate is arrived with the help of MPN table.

$\mathrm{N}$-free semi solid malic acid medium was prepared in tubes of five $\mathrm{mL}$ quantity and sterilize. Ten gram of representative sample (root/rhizosphere soil) was taken and mixed thoroughly in $90 \mathrm{~mL}$ sterile water blank to get $10^{-1}$ dilution from which serial dilutions are prepared up to $10^{-4}$ by transferring one $\mathrm{mL}$ to nine $\mathrm{mL}$ water blank. The dilutions $10^{-2}, 10^{-3}$ and $10^{-4}$ were selected for enumeration. One $\mathrm{ml}$ suspension from each of the dilution was transferred aseptically to each of the five tubes containing the sterilized medium. One set of tubes (5 Nos.) as control without inoculation were maintained and incubated at room temperature $28 \pm 2^{\circ} \mathrm{C}$ for a period of 4 to 6 days.

\section{Estimation of dehydrogenase activity}

Twenty gram of air dried soil $(<2 \mathrm{~mm})$ and 0.2 gram of $\mathrm{CaCO}_{3}$ was thoroughly mixed and place six gram of this mixture in each of three test tubes. Add one $\mathrm{mL}$ of $3 \%$ aqueous solution of triphenyl tetrazolium chloride and $25 \mathrm{~mL}$ of distilled water to each tube. This amount of liquid should be sufficient that a small amount of free liquid appears at the surface of the soil after mixing. The contents 
of each tube with a glass rod were mixed and stopper the tube and incubated it at $37^{\circ} \mathrm{C}$. The stopper was removed after 24 hours, add ten $\mathrm{mL}$ of methanol and stopper the tube and shake it for 1 minute. Tube was unstopper and filtered the suspension through a glass funnel plugged with absorbent cotton into a $100 \mathrm{~mL}$ volumetric flask. Washed the tube with methanol and quantitatively transferred the soil to the funnel, then additional methanol (in $10 \mathrm{~mL}$ portions) was added to the funnel until the reddish color has disappeared from the cotton plug. Filtrate was diluted to a $100 \mathrm{~mL}$ volume with methanol. The intensity of the reddish color was measured by using a spectrophotometer at a wavelength of $485 \mathrm{~nm}$ and a one $\mathrm{cm}$ cuvette with methanol as a blank. The amount of TPF produced was calculated by reference to a calibration graph prepared from TPF standards. To prepare standard graph, diluted ten $\mathrm{mL}$ of Triphenyl Formazon (TPF) standard solution to $100 \mathrm{~mL}$ with methanol (100 $\mathrm{mg}$ of TPF $\mathrm{mL}^{-1}$ ), made up the volume with methanol and mixed thoroughly. The intensity of the red color of TPF as described for the samples was measured. Absorbance readings were plotted against the amount of TPF in the $100 \mathrm{~mL}$ standard solutions.

\section{Results and Discussion}

\section{Effect of different intercropping system on population of soil bacteria}

The different intercropping systems significantly influenced the population of bacteria in both rhizosphere and non rhizosphere soil at 30,60 DAS and at harvest stage.

At 30 DAS, Little millet + blackgram (4:1) recorded significantly higher bacteria population of $205 \times 10^{7} \mathrm{cfu} \mathrm{g}^{-1}$ dry soil and $173 \times 10^{7} \mathrm{cfu} \mathrm{g}^{-1}$ dry soil in both rhizosphere soil and non-rhizosphere soil, respectively. It was on par with little millet + groundnut (4:1) and little millet + greengram (4:1).

Little millet + groundnut $(4: 1)$ recorded significantly higher bacteria population of 301 x $10^{7}$ cfu g $^{-1}$ dry soil and $257 \times 10^{7}$ cfu g $^{-1}$ dry soil in both rhizosphere soil and nonrhizosphere soil, respectively at 60 DAS. It was on par with little millet + blackgram (4:1) and little millet + greengram (4:1).

At harvest stage, Little millet + groundnut (4:1) recorded significantly higher bacteria population of $228 \times 10^{7} \mathrm{cfu} \mathrm{g}^{-1}$ dry soil and $202 \times 10^{7}$ cfu g $^{-1}$ dry soil in both rhizosphere soil and non-rhizosphere soil, respectively. It was on par with little millet + blackgram (4:1) and little millet + greengram (4:1).

The lowest bacteria population were recorded in sole little millet crop in both rhizosphere soil and non-rhizosphere soil at 30, 60 DAS and at harvest stage (Table 1).

\section{Effect of different intercropping system on population of soil fungi}

The different intercropping systems also significantly influenced the population of fungi in both rhizosphere and non rhizosphere soil at 30,60 DAS and at harvest stage.

At 30 DAS, little millet + blackgram (4:1) recorded significantly higher fungi population of $67 \times 10^{4} \mathrm{cfu} \mathrm{g}^{-1}$ dry soil and $64 \times 10^{4} \mathrm{cfu} \mathrm{g}^{-1}$ dry soil in both rhizosphere soil and nonrhizosphere soil, respectively. It was on par with little millet + groundnut (4:1) and little millet + greengram $(4: 1)$.

However, Little millet + groundnut (4:1) had significant fungi population of $75 \times 10^{4} \mathrm{cfu}$ $\mathrm{g}^{-1}$ dry soil and $71 \times 10^{4} \mathrm{cfu} \mathrm{g}^{-1}$ dry soil in both rhizosphere soil and non-rhizosphere soil, respectively at 60 DAS. It was on par with little millet + blackgram (4:1) and little 
millet + greengram (4:1). At harvest stage, Little millet + groundnut $(4: 1)$ recorded significantly higher fungi population of $70 \mathrm{x}$ $10^{4} \mathrm{cfu} \mathrm{g}^{-1}$ dry soil and $67 \times 10^{4} \mathrm{cfu} \mathrm{g}^{-1}$ dry soil in both rhizosphere soil and nonrhizosphere soil respectively at 60 DAS. It was on par with little millet + blackgram (4:1) and little millet + greengram $(4: 1)$.

The lowest fungi population were recorded in sole little millet crop in both rhizosphere soil and non-rhizosphere soil at 30,60 DAS and at harvest stage (Table 2).

\section{Effect of different intercropping system on population of soil actinobacteria}

The different intercropping systems significantly shown the similar trends in population of actinobacteria in both rhizosphere and non rhizosphere soil at 30, 60 DAS and at harvest stage. little millet + blackgram (4:1) recorded significantly higher actinobacteria population of $73 \times 10^{5} \mathrm{cfu} \mathrm{g}^{-1}$ dry soil and $69 \times 10^{5} \mathrm{cfu} \mathrm{g}^{-1}$ dry soil in both rhizosphere soil and non-rhizosphere soil, respectively.

It was on par with little millet + groundnut $(4: 1)$ and little millet + greengram $(4: 1)$ at 30 DAS, At 60 DAS, Little millet + groundnut (4:1) shows significantly higher actinobacteria population of $79 \times 10^{5} \mathrm{cfu} \mathrm{g}^{-1}$ dry soil and $73 \times 10^{5} \mathrm{cfu} \mathrm{g}^{-1}$ dry soil in both rhizosphere soil and non-rhizosphere soil respectively. It was on par with little millet + blackgram (4:1) and little millet + greengram (4:1).

At harvest stage, Little millet + groundnut $(4: 1) \quad$ recorded significantly higher actinobacteria population of $76 \times 10^{5} \mathrm{cfu}^{-1}$ dry soil and $70 \times 10^{5} \mathrm{cfu} \mathrm{g}^{-1}$ dry soil in both rhizosphere soil and non-rhizosphere soil respectively at 60 DAS. It was on par with little millet + blackgram $(4: 1)$ and little millet
+ greengram (4:1). The lowest fungi population were recorded in little millet sole crop in both rhizosphere soil and nonrhizosphere soil at 30,60 DAS and at harvest stage (Table 3).

Daisy et al., (2018) reported that the microbial population such as bacteria, fungi and actinobacteria were significantly superior under legume intercropped systems than sole crop of little millet. Similar findings were reported by Li et al., (2016); Zhang et al., (2017); Rekha et al., (2017); Li and $\mathrm{Wu}$ (2018); Li et al., (2018). This might be due to a greater root biomass and soil $\mathrm{C}$ content of intercropping systems than that of sole crop. Another potential indirect effect in the rhizosphere of intercropped species is enhanced nutrient mineralization due to the change in soil organic matter decomposition rates, resulting from the addition of fresh organic matter (Khan et al., 2014).

Becker and Ladha (1995) and Peoples et al., (1995) reported that the incorporation of legume stubbles under intercropping systems contributed large amount of nitrogen and organic matter to the soil and also favoured for the better proliferation of the soil microorganisms (Table 4).

\section{Effect of different intercropping system on} dehydrogenase enzyme activity of soil bacteria

Dehydrogenase enzyme plays a vital role in the biological oxidation of soil organic matter by transferring hydrogen from organic substrates to inorganic acceptors (Zhang et al., 2010). It is a measure of soil microbial activity (Trevors, 1984; Garcia and Hernandez et al., 1997). Among all enzymes in soil environment, the dehydogenase enzyme activity is commonly used as an indicator of biological activity in soils (Burns, 1978). 
Table.1 Effect of different intercropping system on bacteria population in both rhizosphere soil and non-rhizosphere soil at 30 , 60 DAS and at harvest stage

\begin{tabular}{|c|c|c|c|c|c|c|c|c|c|c|c|}
\hline & \multirow[t]{3}{*}{ Treatments } & \multirow[t]{3}{*}{ Crop } & \multicolumn{9}{|c|}{ Population cfu $\times 10^{7} \mathrm{~g}^{-1}$ of dry weight of soil } \\
\hline & & & \multicolumn{3}{|c|}{30 DAS } & \multicolumn{3}{|c|}{60 DAS } & \multicolumn{3}{|c|}{ At Harvest } \\
\hline & & & $\mathbf{R S}$ & NRS & $\mathbf{R}: \mathbf{S}$ & $\mathbf{R S}$ & NRS & $\mathbf{R}: \mathbf{S}$ & $\mathbf{R S}$ & NRS & $\mathbf{R}: \mathbf{S}$ \\
\hline$T_{1}$ & $\begin{array}{l}\text { Little millet Sole } \\
\text { crop }\end{array}$ & Little millet & $\begin{array}{c}68 \\
(1.833)\end{array}$ & $\begin{array}{c}36 \\
(1.556)\end{array}$ & 1.89:1 & $\begin{array}{c}164 \\
(2.215)\end{array}$ & $\begin{array}{c}120 \\
(2.079)\end{array}$ & $1.37: 1$ & $\begin{array}{c}91 \\
(1.959)\end{array}$ & $\begin{array}{c}65 \\
(1.813)\end{array}$ & 1.40:1 \\
\hline $\mathbf{T}_{2}$ & $\begin{array}{l}\text { Little millet }+ \\
\text { Black gram }(4: 1)\end{array}$ & Black gram & $\begin{array}{c}205 \\
(2.312)\end{array}$ & $\begin{array}{c}173 \\
(2.238)\end{array}$ & $1.18: 1$ & $\begin{array}{c}280 \\
(2.447)\end{array}$ & $\begin{array}{c}236 \\
(2.373)\end{array}$ & $1.19: 1$ & $\begin{array}{c}207 \\
(2.316)\end{array}$ & $\begin{array}{c}181 \\
(2.258)\end{array}$ & $1.14: 1$ \\
\hline $\mathbf{T}_{3}$ & $\begin{array}{l}\text { Little millet }+ \\
\text { Green gram }(4: 1)\end{array}$ & Green gram & $\begin{array}{c}173 \\
(2.238)\end{array}$ & $\begin{array}{c}141 \\
(2.149)\end{array}$ & $1.23: 1$ & $\begin{array}{c}269 \\
(2.430)\end{array}$ & $\begin{array}{c}225 \\
(2.352)\end{array}$ & $1.20: 1$ & $\begin{array}{c}196 \\
(2.292)\end{array}$ & $\begin{array}{c}170 \\
(2.230)\end{array}$ & $1.15: 1$ \\
\hline $\mathbf{T}_{4}$ & $\begin{array}{l}\text { Little millet }+ \\
\text { Cowpea }(4: 1)\end{array}$ & Cowpea & $\begin{array}{c}142 \\
(2.152)\end{array}$ & $\begin{array}{c}110 \\
(2.041)\end{array}$ & 1.29:1 & $\begin{array}{c}238 \\
(2.377)\end{array}$ & $\begin{array}{c}184 \\
(2.265)\end{array}$ & $1.29: 1$ & $\begin{array}{c}165 \\
(2.217)\end{array}$ & $\begin{array}{c}139 \\
(2.143)\end{array}$ & $1.19: 1$ \\
\hline $\mathbf{T}_{5}$ & $\begin{array}{l}\text { Little millet }+ \\
\text { Groundnut }(4: 1)\end{array}$ & Groundnut & $\begin{array}{c}184 \\
(2.265)\end{array}$ & $\begin{array}{c}152 \\
(2.182)\end{array}$ & 1.21:1 & $\begin{array}{c}301 \\
(2.479)\end{array}$ & $\begin{array}{c}257 \\
(2.410)\end{array}$ & $1.17: 1$ & $\begin{array}{c}228 \\
(2.352)\end{array}$ & $\begin{array}{c}202 \\
(2.305)\end{array}$ & $1.13: 1$ \\
\hline$T_{6}$ & $\begin{array}{l}\text { Little millet }+ \\
\text { Horse gram }(4: 1)\end{array}$ & Horsegram & $\begin{array}{c}121 \\
(2.236)\end{array}$ & $\begin{array}{c}89 \\
(1.949)\end{array}$ & $1.36: 1$ & $\begin{array}{c}217 \\
(2.336)\end{array}$ & $\begin{array}{c}138 \\
(2.410)\end{array}$ & $1.57: 1$ & $\begin{array}{c}144 \\
(2.158)\end{array}$ & $\begin{array}{c}118 \\
(2.072)\end{array}$ & $1.22: 1$ \\
\hline $\mathbf{T}_{7}$ & $\begin{array}{l}\text { Little millet }+ \\
\text { Small onion }(4: 1)\end{array}$ & Small onion & $\begin{array}{c}89 \\
(1.949)\end{array}$ & $\begin{array}{c}57 \\
(1.756)\end{array}$ & $1.56: 1$ & $\begin{array}{c}185 \\
(2.267)\end{array}$ & $\begin{array}{c}132 \\
(2.121)\end{array}$ & $1.40: 1$ & $\begin{array}{c}112 \\
(2.049)\end{array}$ & $\begin{array}{c}82 \\
(1.914)\end{array}$ & $1.37: 1$ \\
\hline $\mathbf{T}_{8}$ & $\begin{array}{l}\text { Little millet }+ \\
\text { Coriander }(4: 1)\end{array}$ & Coriander & $\begin{array}{c}92 \\
(1.964)\end{array}$ & $\begin{array}{c}60 \\
(1.778)\end{array}$ & $1.53: 1$ & $\begin{array}{c}188 \\
(2.274)\end{array}$ & $\begin{array}{c}126 \\
(2.100)\end{array}$ & $1.49: 1$ & $\begin{array}{c}115 \\
(2.061)\end{array}$ & $\begin{array}{c}85 \\
(1.929)\end{array}$ & $1.35: 1$ \\
\hline \multicolumn{3}{|c|}{ SEd } & 0.011 & 0.084 & & 0.065 & 0.063 & & 0.060 & 0.057 & \\
\hline \multicolumn{3}{|c|}{$C D(p=0.05)$} & 0.025 & 0.184 & & 0.140 & 0.135 & & 0.130 & 0.124 & \\
\hline
\end{tabular}

Figure in parenthesis are logarithmic transformed values.

RS-Rhizosphere Soil; NRS- Non-Rhizosphere Soil; R:S- Ratio of Rhizosphere Soil and Non-Rhizosphere Soil 
Table.2 Effect of different intercropping system on fungi population in both rhizosphere soil and non-rhizosphere soil at 30,60 DAS and at harvest stage

\begin{tabular}{|c|c|c|c|c|c|c|c|c|c|c|c|}
\hline & \multirow[t]{3}{*}{ Treatments } & \multirow[t]{3}{*}{ Crop } & \multicolumn{9}{|c|}{ Population cfu $\times 10^{4} \mathrm{~g}^{-1}$ of dry weight of soil } \\
\hline & & & \multicolumn{3}{|c|}{30 DAS } & \multicolumn{3}{|c|}{60 DAS } & \multicolumn{3}{|c|}{ At Harvest } \\
\hline & & & $\mathbf{R S}$ & NRS & $\mathbf{R : S}$ & $\mathbf{R S}$ & NRS & $\mathbf{R : S}$ & $\mathbf{R S}$ & NRS & R:S \\
\hline $\mathbf{T}_{1}$ & $\begin{array}{l}\text { Little millet Sole } \\
\text { crop }\end{array}$ & Little millet & $\begin{array}{c}13 \\
(1.114)\end{array}$ & $\begin{array}{c}10 \\
(1.000)\end{array}$ & $1.30: 1$ & $\begin{array}{c}21 \\
(1.322)\end{array}$ & $\begin{array}{c}15 \\
(1.176)\end{array}$ & 1.40:1 & $\begin{array}{c}16 \\
(1.204)\end{array}$ & $\begin{array}{c}13 \\
(1.114)\end{array}$ & $1.23: 1$ \\
\hline $\mathbf{T}_{2}$ & $\begin{array}{l}\text { Little millet }+ \\
\text { Black gram }(4: 1)\end{array}$ & Black gram & $\begin{array}{c}67 \\
(1.826)\end{array}$ & $\begin{array}{c}64 \\
(1.806)\end{array}$ & $1.05: 1$ & $\begin{array}{c}72 \\
(1.857)\end{array}$ & $\begin{array}{c}70 \\
(1.845)\end{array}$ & 1.03:1 & $\begin{array}{c}67 \\
(1.826)\end{array}$ & $\begin{array}{c}62 \\
(1.792)\end{array}$ & 1.08:1 \\
\hline $\mathbf{T}_{3}$ & $\begin{array}{l}\text { Little millet }+ \\
\text { Green gram }(4: 1)\end{array}$ & Green gram & $\begin{array}{c}55 \\
(1.740)\end{array}$ & $\begin{array}{c}53 \\
(1.724)\end{array}$ & $1.04: 1$ & $\begin{array}{c}61 \\
(1.785)\end{array}$ & $\begin{array}{c}69 \\
(1.839)\end{array}$ & $0.88: 1$ & $\begin{array}{c}62 \\
(1.792)\end{array}$ & $\begin{array}{c}61 \\
(1.785)\end{array}$ & $1.02: 1$ \\
\hline $\mathbf{T}_{4}$ & $\begin{array}{l}\text { Little millet }+ \\
\text { Cowpea }(4: 1)\end{array}$ & Cowpea & $\begin{array}{c}43 \\
(1.633)\end{array}$ & $\begin{array}{c}40 \\
(1.602)\end{array}$ & $1.08: 1$ & $\begin{array}{c}51 \\
(1.708)\end{array}$ & $\begin{array}{c}45 \\
(1.653)\end{array}$ & $1.13: 1$ & $\begin{array}{c}46 \\
(1.663)\end{array}$ & $\begin{array}{c}43 \\
(1.633)\end{array}$ & $1.07: 1$ \\
\hline $\mathbf{T}_{5}$ & $\begin{array}{l}\text { Little millet }+ \\
\text { Groundnut }(4: 1)\end{array}$ & Groundnut & $\begin{array}{c}60 \\
(1.778)\end{array}$ & $\begin{array}{c}57 \\
(1.756)\end{array}$ & $1.05: 1$ & $\begin{array}{c}75 \\
(1.875)\end{array}$ & $\begin{array}{c}71 \\
(1.851)\end{array}$ & 1.06:1 & $\begin{array}{c}70 \\
(1.845)\end{array}$ & $\begin{array}{c}67 \\
(1.826)\end{array}$ & $1.04: 1$ \\
\hline$T_{6}$ & $\begin{array}{l}\text { Little millet }+ \\
\text { Horse gram }(4: 1)\end{array}$ & Horsegram & $\begin{array}{c}40 \\
(1.602)\end{array}$ & $\begin{array}{c}37 \\
(1.568)\end{array}$ & 1.08:1 & $\begin{array}{c}48 \\
(1.681)\end{array}$ & $\begin{array}{c}42 \\
(1.623)\end{array}$ & $1.14: 1$ & $\begin{array}{c}43 \\
(1.633)\end{array}$ & $\begin{array}{c}40 \\
(1.602)\end{array}$ & 1.08:1 \\
\hline $\mathbf{T}_{7}$ & $\begin{array}{l}\text { Little millet }+ \\
\text { Small onion }(4: 1)\end{array}$ & Small onion & $\begin{array}{c}14 \\
(1.146)\end{array}$ & $\begin{array}{c}11 \\
(1.041)\end{array}$ & $1.27: 1$ & $\begin{array}{c}22 \\
(1.342)\end{array}$ & $\begin{array}{c}16 \\
(1.204)\end{array}$ & $1.38: 1$ & $\begin{array}{c}17 \\
(1.230)\end{array}$ & $\begin{array}{c}14 \\
(1.146)\end{array}$ & $1.21: 1$ \\
\hline $\mathbf{T}_{8}$ & $\begin{array}{l}\text { Little millet }+ \\
\text { Coriander }(4: 1)\end{array}$ & Coriander & $\begin{array}{c}12 \\
(1.176)\end{array}$ & $\begin{array}{c}12 \\
(1.079)\end{array}$ & $1.25: 1$ & $\begin{array}{c}26 \\
(1.415)\end{array}$ & $\begin{array}{c}17 \\
(1.203)\end{array}$ & $1.53: 1$ & $\begin{array}{c}19 \\
(1.247)\end{array}$ & $\begin{array}{c}15 \\
(1.176)\end{array}$ & $1.27: 1$ \\
\hline \multicolumn{3}{|c|}{ SEd } & 0.041 & 0.038 & & 0.044 & 0.042 & & 0.042 & 0.041 & \\
\hline \multicolumn{3}{|c|}{$\mathrm{CD}(\mathrm{p}=0.05)$} & 0.088 & 0.083 & & 0.096 & 0.091 & & 0.092 & 0.088 & \\
\hline
\end{tabular}

Figure in parenthesis are logarithmic transformed values.

RS-Rhizosphere Soil; NRS- Non-Rhizosphere Soil; R:S- Ratio of Rhizosphere Soil and Non-Rhizosphere Soil 
Table.3 Effect of different intercropping system on actinobacteria population in both rhizosphere soil and non-rhizosphere soil at 30,60 DAS and at harvest stage

\begin{tabular}{|c|c|c|c|c|c|c|c|c|c|c|c|}
\hline & \multirow[t]{3}{*}{ Treatments } & \multirow[t]{3}{*}{ Crop } & \multicolumn{9}{|c|}{ Population cfu $\times 10^{5} \mathrm{~g}^{-1}$ of dry weight of soil } \\
\hline & & & \multicolumn{3}{|c|}{30 DAS } & \multicolumn{3}{|c|}{60 DAS } & \multicolumn{3}{|c|}{ At Harvest } \\
\hline & & & $\mathbf{R S}$ & NRS & $\mathbf{R : S}$ & $\mathbf{R S}$ & NRS & $\mathbf{R : S}$ & $\mathbf{R S}$ & NRS & R:S \\
\hline$T_{1}$ & $\begin{array}{l}\text { Little millet Sole } \\
\text { crop }\end{array}$ & Little millet & $\begin{array}{c}19 \\
(1.279)\end{array}$ & $\begin{array}{c}15 \\
(1.176)\end{array}$ & $1.27: 1$ & $\begin{array}{c}25 \\
(1.398)\end{array}$ & $\begin{array}{c}19 \\
(1.279)\end{array}$ & $1.32: 1$ & $\begin{array}{c}22 \\
(1.342)\end{array}$ & $\begin{array}{c}16 \\
(1.204)\end{array}$ & 1.38:1 \\
\hline $\mathbf{T}_{2}$ & $\begin{array}{l}\text { Little millet }+ \\
\text { Black gram }(4: 1)\end{array}$ & Black gram & $\begin{array}{c}73 \\
(1.863)\end{array}$ & $\begin{array}{c}69 \\
(1.839)\end{array}$ & $1.06: 1$ & $\begin{array}{c}76 \\
(1.881)\end{array}$ & $\begin{array}{c}71 \\
(1.851)\end{array}$ & 1.07:1 & $\begin{array}{c}73 \\
(1.863)\end{array}$ & $\begin{array}{c}68 \\
(1.833)\end{array}$ & $1.07: 1$ \\
\hline $\mathbf{T}_{3}$ & $\begin{array}{l}\text { Little millet }+ \\
\text { Green gram }(4: 1)\end{array}$ & Green gram & $\begin{array}{c}59 \\
(1.771)\end{array}$ & $\begin{array}{c}56 \\
(1.748)\end{array}$ & $1.05: 1$ & $\begin{array}{c}58 \\
(1.763)\end{array}$ & $\begin{array}{c}59 \\
(1.771)\end{array}$ & 0.98:1 & $\begin{array}{c}55 \\
(1.470)\end{array}$ & $\begin{array}{c}56 \\
(1.748)\end{array}$ & 0.98:1 \\
\hline $\mathbf{T}_{4}$ & $\begin{array}{l}\text { Little millet }+ \\
\text { Cowpea }(4: 1)\end{array}$ & Cowpea & $\begin{array}{c}49 \\
(1.690)\end{array}$ & $\begin{array}{c}45 \\
(1.653)\end{array}$ & 1.09:1 & $\begin{array}{c}55 \\
(1.740)\end{array}$ & $\begin{array}{c}49 \\
(1.690)\end{array}$ & $1.12: 1$ & $\begin{array}{c}52 \\
(1.716)\end{array}$ & $\begin{array}{c}46 \\
(1.663)\end{array}$ & 1.13:1 \\
\hline $\mathbf{T}_{5}$ & $\begin{array}{l}\text { Little millet }+ \\
\text { Groundnut }(4: 1)\end{array}$ & Groundnut & $\begin{array}{c}66 \\
(1.820)\end{array}$ & $\begin{array}{c}62 \\
(1.792)\end{array}$ & $1.06: 1$ & $\begin{array}{c}79 \\
(1.898)\end{array}$ & $\begin{array}{c}73 \\
(1.863)\end{array}$ & 1.08:1 & $\begin{array}{c}76 \\
(1.881)\end{array}$ & $\begin{array}{c}70 \\
(1.845)\end{array}$ & 1.09:1 \\
\hline$T_{6}$ & $\begin{array}{l}\text { Little millet }+ \\
\text { Horse gram }(4: 1)\end{array}$ & Horsegram & $\begin{array}{c}46 \\
(1.663)\end{array}$ & $\begin{array}{c}42 \\
(1.623)\end{array}$ & $1.10: 1$ & $\begin{array}{c}52 \\
(1.716)\end{array}$ & $\begin{array}{c}46 \\
(1.663)\end{array}$ & $1.13: 1$ & $\begin{array}{c}49 \\
(1.690)\end{array}$ & $\begin{array}{c}43 \\
(1.663)\end{array}$ & $1.14: 1$ \\
\hline $\mathbf{T}_{7}$ & $\begin{array}{l}\text { Little millet }+ \\
\text { Small onion }(4: 1)\end{array}$ & Small onion & $\begin{array}{c}20 \\
(1.301)\end{array}$ & $\begin{array}{c}16 \\
(1.204)\end{array}$ & $1.25: 1$ & $\begin{array}{c}26 \\
(1.415)\end{array}$ & $\begin{array}{c}20 \\
(1.301)\end{array}$ & $1.30: 1$ & $\begin{array}{c}23 \\
(1.362)\end{array}$ & $\begin{array}{c}17 \\
(1.230)\end{array}$ & $1.35: 1$ \\
\hline $\mathbf{T}_{8}$ & $\begin{array}{l}\text { Little millet }+ \\
\text { Coriander }(4: 1)\end{array}$ & Coriander & $\begin{array}{c}24 \\
(1.380)\end{array}$ & $\begin{array}{c}17 \\
(1.203)\end{array}$ & $1.41: 1$ & $\begin{array}{c}30 \\
(1.477)\end{array}$ & $\begin{array}{c}21 \\
(1.322)\end{array}$ & $1.43: 1$ & $\begin{array}{c}27 \\
(1.431)\end{array}$ & $\begin{array}{c}18 \\
(1.255)\end{array}$ & $1.50: 1$ \\
\hline \multicolumn{3}{|c|}{ SEd } & 0.044 & 0.043 & & 0.046 & 0.044 & & 0.045 & 0.042 & \\
\hline \multicolumn{3}{|c|}{ CD $(p=0.05)$} & 0.094 & 0.092 & & 0.099 & 0.094 & & 0.094 & 0.091 & \\
\hline
\end{tabular}

Figure in parenthesis are logarithmic transformed values.

RS-Rhizosphere Soil; NRS- Non-Rhizosphere Soil; R:S- Ratio of Rhizosphere Soil and Non-Rhizosphere Soil 
Table.4 Effect of different intercropping system on postharvest soil fertility status

\begin{tabular}{|c|l|c|c|c|}
\hline & \multicolumn{1}{|c|}{ Treatments } & Available N & Available P & Available K \\
\hline $\mathbf{T}_{\mathbf{1}}$ & Little millet Sole crop & 216.8 & 17.8 & 250.2 \\
\hline $\mathbf{T}_{\mathbf{2}}$ & Little millet + Black gram (4:1) & 237.7 & 21.7 & 274.5 \\
\hline $\mathbf{T}_{\mathbf{3}}$ & Little millet + Green gram (4:1) & 236.5 & 20.7 & 272.3 \\
\hline $\mathbf{T}_{\mathbf{4}}$ & Little millet + Cowpea (4:1) & 235.5 & 20.5 & 271.8 \\
\hline $\mathbf{T}_{\mathbf{5}}$ & Little millet + Groundnut (4:1) & 234.2 & 20.2 & 264.2 \\
\hline $\mathbf{T}_{\mathbf{6}}$ & Little millet + Horse gram (4:1) & 234.9 & 20.1 & 268.7 \\
\hline $\mathbf{T}_{\mathbf{7}}$ & Little millet + Small onion (4:1) & 216.3 & 17.3 & 246.3 \\
\hline $\mathbf{T}_{\mathbf{8}}$ & Little millet + Coriander (4:1) & 215.1 & 16.2 & 241.6 \\
\hline & $\mathbf{S E d}$ & 9.43 & 0.79 & 10.8 \\
\hline & $\mathbf{C D}(\mathbf{p = 0 . 0 5 )}$ & 20.38 & 1.71 & 23.2 \\
\hline
\end{tabular}

Fig.1\&2 Bulk soil - Hand shaking vigorously for $10 \mathrm{~min}$ and Rhizosphere soil hand shaking 1L $\mathrm{NaCl} 0.9 \%$ for $10 \mathrm{~min}$
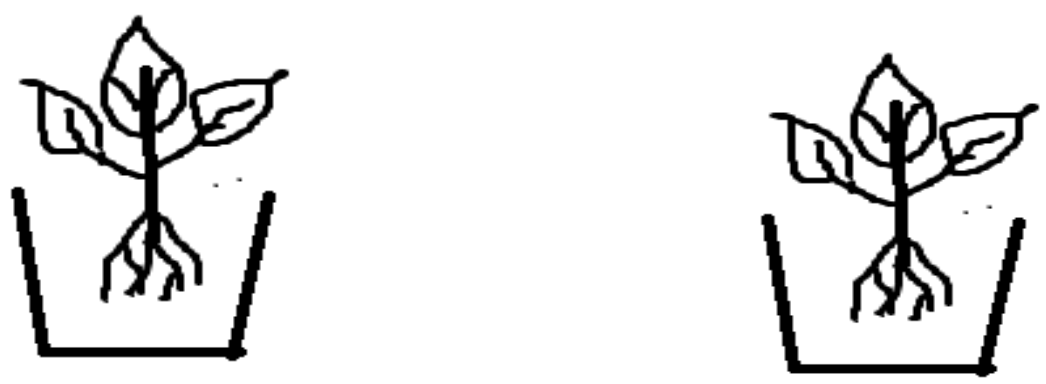

Fig.3

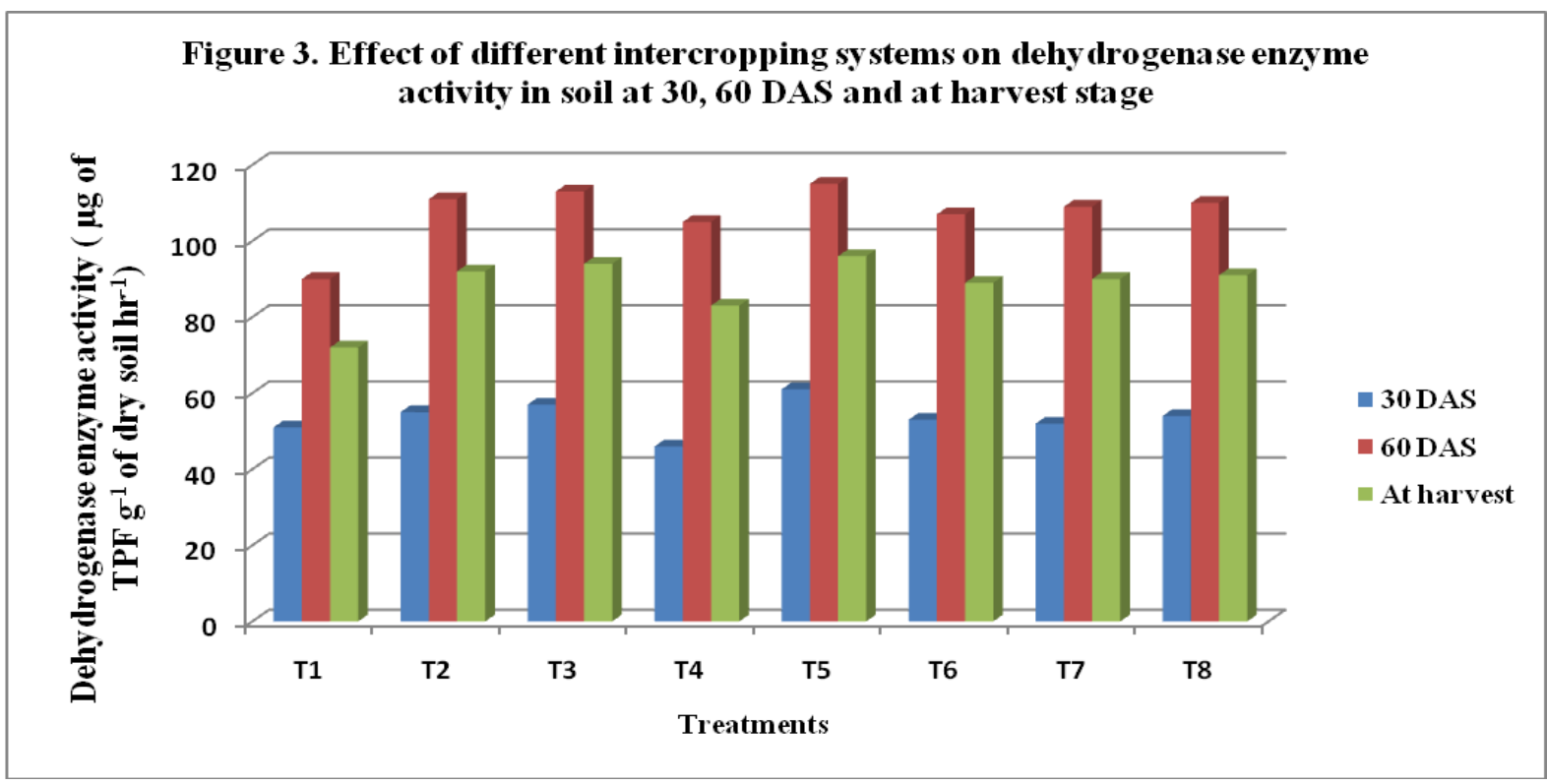


Fig.4

\section{Figure 4: Effect of different intercropping sy stems on Azospirillum population} in soil at 30, 60 DAS and at harvest stage

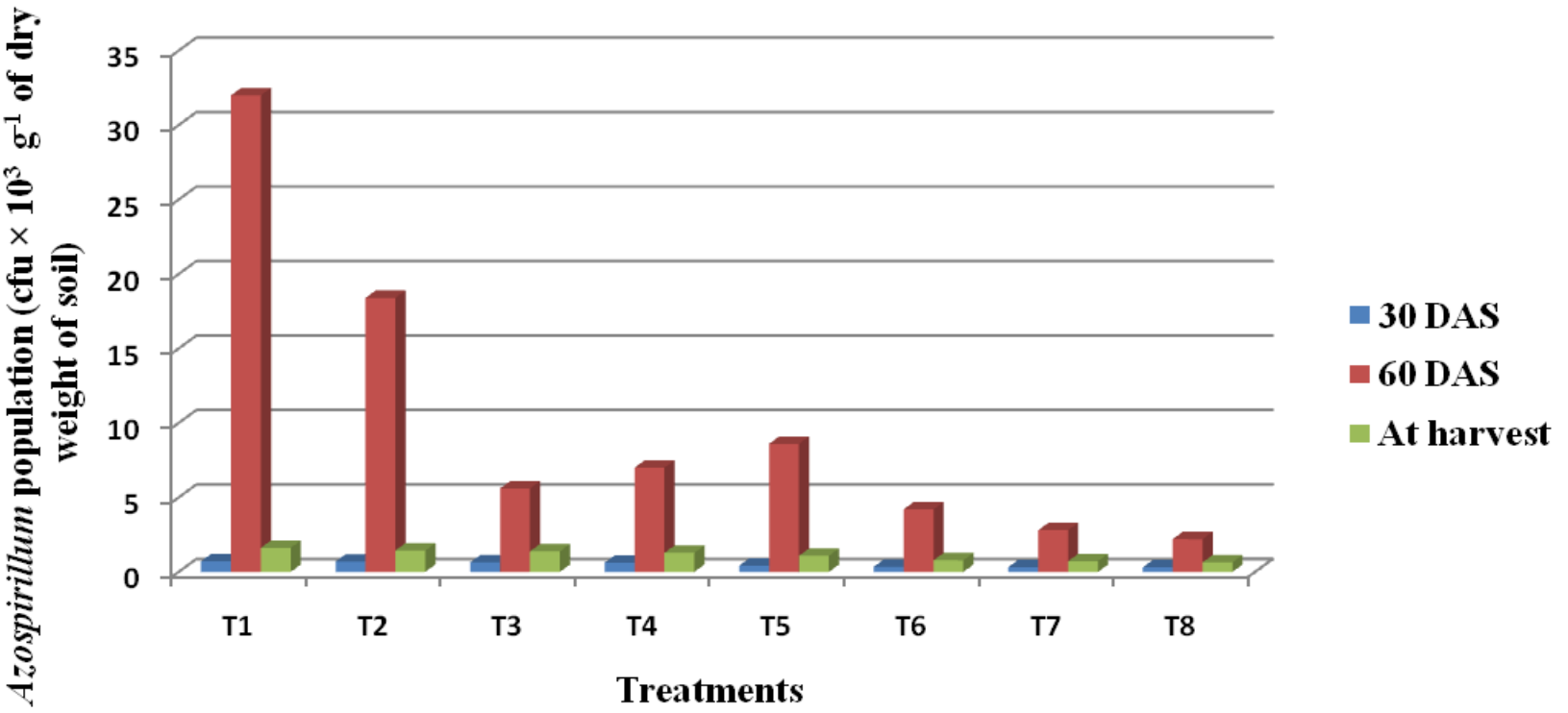

The various intercropping systems significantly influenced the dehydrogenase enzyme activity in soil at 30,60 DAS and harvest stage.

In general, little millet + groundnut $(4: 1)$ recorded higher dehydrogenase enzyme activity of 61,115 and $96 \mu \mathrm{g}$ of TPF $\mathrm{g}^{-1}$ of dry soil $\mathrm{hr}^{-1}$ at 30,60 DAS and harvest stage, respectively. The lowest dehydrogenase enzyme activity of 51,90 and $72 \mu \mathrm{g}$ of TPF $\mathrm{g}^{-1}$ of dry soil $\mathrm{hr}^{-1}$ at 30, 60 DAS and harvest stage, respectively were observed in sole little millet (Figure 3).

Jiang et al., (2010) who found that intercropping system could improve the soil enzyme activity. Similar findings were also reported by (Xingang et al., 2011). Improvement in soil quality by the green manure legumes was reported by Shah et al., (2011). Cai et al., (2010) who reported that enzyme activities were enhanced significantly after intercropping. These findings are also supported by Yang and Fz (2011) and Han et al., (2013).

\section{Effect of different intercropping system on population of soil Azospirillum}

On the controversy of above results, Azospirillum population showed a reverse trends. Little millet sole crop recorded higher Azospirillum population of $0.72,32$ and 1.62 MPN (x $10^{3}$ cells $\mathrm{g}^{-1}$ of dry soil) at 30,60 DAS and at harvest stages, respectively and it was followed by the association of little millet + blackgram (4:1). The lowest values on Azospirillum population $(0.31,2.2$ and 0.64 MPN) (x $10^{3}$ cells $\mathrm{g}^{-1}$ of dry soil) at 30,60 DAS and harvest stages, respectively were evidenced in little millet + coriander $(4: 1)$ (Figure 4). The Azospirillum population were higher in little millet sole crop than the 
intercropping system. These findings were reported by Mohammed Rafi and Charyulu (2010).

In conclusion, the microbial population of soil bacteria, fungi, actinobacteria and dehydrogenase enzyme activity were higher in the little millet based legume intercropping system as it correlated with higher postharvest soil fertility status like available nitrogen, phosphorus and potassium compared to sole crop of little millet. Hence, compared to sole crop of little millet, intercropping of little millet + groundnut $(4: 1)$, little millet + black gram (4:1) and little millet + green gram (4:1) were selected as best intercropping system for Tamirabarani command area.

\section{References}

Anonymous. 2016. Agricultural census, Directorate of economics and Statistics, Department of Agriculture and Cooperation, Government of India.

Ahmad, I., Cheng, Z., Meng, H., Liu, T., Wang, M., Ejaz, M., and Wasila, H. (2013). Effect of pepper-garlic intercropping system on soil microbial and bio-chemical properties. Pak. J. Bot, 45(2), 695-702.

Becker, M. and J. K. Ladha. 1995. Green manure technology. Potential usage and limitations. Plant and Soil. 174.

Burns RG. Enzyme activity in soil: some theoretical and practical considerations. In: Bum RG (ed) Soil enzymes. Academic, London, 1978, 295-340.

Daisy, M., K. Rajendran and Mohamed Amanullah, M. 2018. Effect on Microbial Population, Quality Parameters and Green Fodder Yield of Leguminous Crops under Bt Cotton Intercropping System. Int.J.Curr. Microbiol.App.Sci. 7(01): 332-337.

Dahmardeh, M., Ghanbari, A., Syahsar, B. A., and Ramrodi, M. (2010). The role of intercropping maize (Zea mays L.) and cowpea (Vigna unguiculata L.) on yield and soil chemical properties. African Journal of Agricultural Research, 5(8), 631-636.

Dubey, O. P., and Shrivas, D. N. (1997). Productivity and economics of kodo millet (Paspalum scrobiculatum)-based intercropping systems under rainfed conditions. Indian Journal of Agronomy, 42(2), 224-227.

Garcia C, Hernandez T. Biological and biochemical indicators in derelict soils subject to erosion. Soil Biol Biochem. 1997; 29:171-177.

Himasree, B., Chandrika, V., Sarala, N. V., and Prasanthi, A. (2017). Evaluation of remunerative foxtail millet (Setaria italica L.) based intercropping systems under late sown conditions.

Khan MA, Chen J, Qisong L, Zhang W, Linkun W, Zhenfang L, et al., Effect of interspecific root interaction on soil nutrition, enzymatic activity and rhizosphere biology in maize/peanut intercropping system. Pakistan J. Agri. Sci. 2014; 51(2):395-406.

LI, Q. S., WU, L. K., Jun, C. H. E. N., Khan, M. A., LUO, X. M., and LIN, W. X. (2016). Biochemical and microbial properties of rhizospheres under maize/peanut intercropping. Journal of integrative agriculture, 15(1), 101-110.

Li, Q., Chen, J., Wu, L., Luo, X., Li, N., Arafat, Y., and Lin, W. (2018). Belowground interactions impact the soil bacterial community, soil fertility, and crop yield in maize/peanut intercropping systems. International journal of molecular sciences, 19(2), 622.

Mohammed Rafi $\mathrm{M}$ and Charyulu P.B.B.N. Population dynamics of Azospirillum spp. in the rhizosphere of foxtail millet (Setaria italica (L.) Beauv.). Current Biotica, vol 4 Issue 1,2010. 
Navnage, N. P., Patle, P. N., and Ramteke, P. R. (2018). Dehydrogenase activity (DHA): Measure of total microbial activity and as indicator of soil quality. IJCS, 6(1), 456-458.

Peoples, M.B., D.F. Herridge and J.K. Ladha. 1995. Biological nitrogen fixation an efficient source of nitrogen foe sustainable agriculture. Plant and Soil, Pp. 174.

Rekha, R. G. (2017). Soil microflora as influenced by different intercropping systems and nitrogen management practices. Journal of Pharmacognosy and Phytochemistry, 6(5), 889-891.

Sharmili, K., and Manoharan, S. (2018). Studies on Intercropping in Rainfed Little Millet (Panicum sumatrense). International Journal of Current Microbiology and Applied Sciences, 7(2), 323-327.

Trevors JT. Dehydrogenase activity in soil: a comparison between the INT and TTC assay. Soil Biol Biochem. 1984; 16: 673-674.

Zhang N, He X, Gao Y, Li Y, Wang H, Ma D, Zhang R, Yang S. Pedogenic Carbonate and Soil Dehydrogenase Activity in Response to Soil Organic Matter in Artemisia ordosica Community. Pedosphere. 2010; 20: 229-235.

Zhang, M. M., Wang, N., Hu, Y. B., and Sun, G. Y. (2018). Changes in soil physicochemical properties and soil bacterial community in mulberry (Morus alba L.)/alfalfa (Medicago sativa L.) intercropping system. Microbiology Open, 7(2), e00555.

Zhou, X., Yu, G., and Wu, F. (2011). Effects of intercropping cucumber with onion or garlic on soil enzyme activities, microbial communities and cucumber yield. European Journal of Soil Biology, 47(5), 279-287.

\section{How to cite this article:}

Keerthanapriya, S., M. Hemalatha, S.P. Ramanathan and Jeberlin Prabina, B. 2019. Studies on Microbial Dynamics in Little Millet (Panicum sumatrense L.) based Intercropping System under Rainfed Condition. Int.J.Curr.Microbiol.App.Sci. 8(06): 819-830. doi: https://doi.org/10.20546/ijcmas.2019.806.099 